\title{
Practical Provably Secure Multi-node Communication
}

\author{
Omar Ali \\ Wireless Research Center \\ E-JUST, Egypt \\ omar.ali@ejust.edu.eg
}

\author{
Mahmoud F. Ayoub \\ Dept. of Comp. and Sys. Eng. \\ Alexandria Univ., Egypt \\ mfayoub@alexu.edu.eg
}

\author{
Moustafa Youssef \\ Wireless Research Center \\ E-JUST, Egypt \\ moustafa.youssef@ejust.edu.eg
}

\begin{abstract}
We present a practical and provably-secure multinode communication scheme in the presence of a passive eavesdropper. The scheme is based on a random scheduling approach that hides the identity of the transmitter from the eavesdropper. This random scheduling leads to ambiguity at the eavesdropper with regard to the origin of the transmitted frame. We present the details of the technique and analyze it to quantify the secrecyfairness-overhead trade-off. Implementation of the scheme over Crossbow Telosb motes, equipped with $\mathrm{CC2420}$ radio chips, shows that the scheme can achieve significant secrecy gain with vanishing outage probability. In addition, it has significant overhead advantage over direct extensions to two-nodes schemes. The technique also has the advantage of allowing inactive nodes to leverage sleep mode to further save energy.
\end{abstract}

\section{INTRODUCTION}

With the continuous growth of wireless networks and emerging new technologies such as WiMAX and LTE, wireless networks security has received extensive attention. Current popular security schemes, e.g. public key cryptography, are based on computationally secure trapdoor one-way functions [1]. These schemes depend on the assumption that it is hard for an attacker to decipher the message without knowing the trapdoor (i.e. the secret key). However, these schemes do not prevent a computationally unlimited attacker from decrypting the message without knowing the trapdoor as it is not proven yet that one-way functions cannot be inverted efficiently [1]. Therefore, these schemes are not provably secure.

Information theoretic secrecy, on the other hand, introduces the possibility of having perfectly secure communication independently from the computational capabilities of the attacker [2]-[15]. In particular, Shannon [2] proved that, using a shared secret key $K$, the achievability of perfect secrecy requires that the entropy of $K$ be at least equal to the entropy of the message $M$ (i.e., $H(K) \geq H(M)$ ). Wyner showed that it is possible to send perfectly secure messages at a non-zero rate, without relying on secret keys or any limiting assumptions on the computational power of the wiretapper, under the condition that the source-wiretapper channel is a degraded version of the source-destination channel [3]. This was later extended to the non-degraded scenario in [4]. In [5], [6], the effect of fading on the secrecy capacity was studied and it was shown that distributing the message across different fading realizations actually increases the secrecy capacity.

Although information theoretic security schemes provide provable security, they have been considered not practical due to the simplifying assumptions they have to prove their security. Recently, we have introduced a number of practical and provably-secure protocols for two-node communication based on information theoretic concepts. Our work in [7][10] exploits the multi-path nature of the wireless medium to provide practical information-theoretic security in channels with feedback. The basic idea is to distribute the secret key among multiple ARQ frames. This concept has been used to enhance the security of practical Wi-Fi and RFID protocols at the expense of slight loss in throughput.

Direct extensions of these two-node schemes to the multinode case, by applying the protocol to each pair of communicating nodes, lead to a considerable waste of throughput. This is due to optimizing each pair independently, extending the two-node overhead to the multi-node case.

In this paper, we present a practical and provably-secure scheme at the presence of a passive eavesdropper that is designed for the multi-node case from the beginning. Our scheme is based on a novel two-phase approach: in the first phase, i.e. the selection phase, a node is selected as the transmitter using information theoretic techniques that hide the identity of the selected node. In the second phase, i.e. the data transmission phase, data frames are transmitted without the source/destination ID in the packet header. This leads to ambiguity at the eavesdropper. The length of the data transmission phase can be tuned to trade-off secrecy and efficiency. Nodes not selected at the selection phase can sleep to the next cycle, further reducing their energy consumption. We present different variations of the basic scheme, all having the same overhead, that can achieve different secrecy-fairness trade-offs. We evaluate our proposed schemes both analytically and through implementation over Crossbow Telosb motes, equipped with CC2420 radio chips. Our evaluation shows that the scheme can achieve both significant secrecy gain and decrease in overhead as compared to direct extensions to the two-node schemes.

The rest of the paper is organized as follows: We define the system model in Section [II Section [II presents the basic scheme. In Section [V] we present four different extensions to the basic scheme that can achieve different secrecy-fairness trade-offs. We analyze the proposed schemes in Section V along with the system implementation. We finally conclude 


\begin{tabular}{|c|l|l|}
\hline Par. & Meaning & Default val. \\
\hline \hline$n$ & Number of network nodes. & 8 \\
\hline$f$ & Num. of packets transm. in a single session. & 4 \\
\hline$l$ & Frame length. & 1024 \\
\hline$k$ & Num. of frames needed to reconstruct orig. message. & 32 \\
\hline$t$ & preamble length & 6 \\
\hline
\end{tabular}

TABLE I

SYMBOLS USED IN THE PAPER.

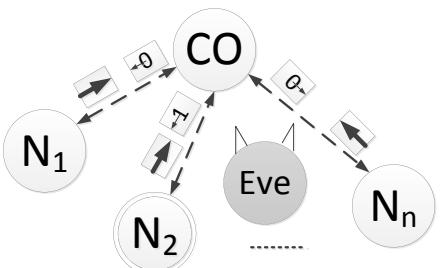

Fig. 1. Network model. A star topology is assumed where each node have to send/receive through the coordinator (CO). In the selection phase, a node is selected if it receives a 1 bit from the $\mathrm{CO}$ using dialog $\operatorname{codes}\left(N_{2}\right.$ in this example).

the paper in Section VI

\section{SySTEM MODEL}

We consider a network with $n$ legitimate nodes in the presence of a passive eavesdropper (Eve). We assume a star topology, where all the traffic between nodes has to go through a central node, i.e. a coordinator. This is common in WLANs, cellular, and sensor network 1]. This coordinator (e.g. access point, base station, or gateway) is responsible for controlling the transmission in the network and assigning turns. All nodes are equipped with half-duplex antennas. We further assume a time-slotted communication system, where all nodes are synchronized (Figure 11).

For space constraints, we also assume that all nodes have equal load and Eve cannot differentiate between nodes based on powert We leave the general case to a future paper.

To further remove the need of acknowledgment, each message $M$ is erasure-coded into $m$ frames such that the reception of any $k<m$ frames at the receiver can be used to reconstitute $M$ with high probability. Note that using erasure coding does not give any advantage to Eve as (a) she cannot determine the identity of the transmitter and (b) there is no message level error detection (only CRC at the frame level).

All system parameters are assumed to be known to the eavesdropper along with the details of the technique, but not the instantaneous random values.

Each node needs to send and receive $k$ frames, each of $l$ bits. Table I summarizes the different symbols we use in the paper.

\footnotetext{
${ }^{1}$ Note that a coordinator can also be selected in a distributed manner if no central node is available.

${ }^{2}$ Power randomization can be used in this case to confuse Eve as in our previous work for two nodes [11].
}

\section{BASIC SCHEME}

The basic idea of our approach is to hide the identity of the packet receiver at any point in time from the eavesdropper. This creates an ambiguity that gives advantage to legitimate nodes. In particular, the system works in two phases: a receiver selection phase and a data transmission phase. In the first phase, the coordinator (CO) informs one of the $n$ nodes securely, using physical layer security principles, that it is selected as the receiver for the packets in the second phase. In the data transmission phase, only the intended receiver has to be active, all other nodes can go to sleep mode, reducing the energy consumption of other nodes. An eavesdropper receiving a packet cannot determine the destination of the packet, and hence has to guess.

The selection phase can be repeated as frequently as needed, by reducing the number of frames during the data transmission phase $(f)$, to increase security. However, this increases the system overhead. Therefore, we have a tradeoff between secrecy and overhead.

In the rest of this section, we present the details of the two phases, assuming one way communication from the coordinator to the nodes. We present the two-way case in Section IV and analyze the overhead and security of the system in Section $\mathrm{V}$

\section{A. Receiver Selection Phase}

During this phase, the coordinator (CO) selects one of the $n$ nodes to be active during the data transmission phase. This is achieved by sending a one bit from the coordinator to each node indicating whether this node is selected (bit=1) or not (bit=0). We adopt dialog codes [12] as the provably secure technique for exchanging these bits. In perfect conditions, dialog codes encode each bit as two bits and the receiver jams only one of these two bits randomly. Under a binary modulo-2 additive channel model, Eve has to guess which bit has been jammed, while Bob knows the correct bit with no ambiguity.

To accommodate realistic situations, [12] adds a $t-1$ bit randomly chosen preamble to the source bit and then encodes the result by dialog codes. The probability of Eve correctly guessing the transmitted bit $\left(P_{E}\right)$ then becomes:

$$
P_{E}=\frac{1}{2}\left(1+(1-w)^{\frac{t+1}{2}}\right), w=\min (p, q)
$$

where $p$ and $q$ are the probability of corrupting 0 to 1 and 1 to 0 respectively.

Equation 11 shows that $P_{E}$ converges to $\frac{1}{2}$ as $t$ increases. [12] showed that, in practice, the convergence speed is much faster than $\frac{1}{2}\left(1+(1-w)^{\frac{t+1}{2}}\right)$. In addition, they showed that for a typical environment, a 6 to 8 bit preamble is enough to confuse Eve. This has been confirmed in our experiment.

\section{B. Data Transmission Phase}

During this phase, the selected node can send/receive $f$ data frames, where $f$ is a parameter that can be used to tune security versus overhead. Only the selected node needs to be active. Transmitted frames do not contain the ID of the 
receiver, which leads to ambiguity at the eavesdropper about the origin of the frames.

\section{TWO-WAY COMMUNICATION}

To allow for two-way communication, we need to specify which slots within a session in the data transmission phase will be from/to the coordinator. In order to do that, we add a third short direction determination phase between the selection and data transmission phases in which the CO sends $f$ bits, using dialog codes again, where each bit corresponds to a slot in the data transmission session. A bit set to 1 (0) corresponds to a from (to) $\mathrm{CO}$ slot. Note that the node ID is not sent in this phase. Therefore, Eve cannot know the identity of the selected node.

In the data transmission phase, a node will follow the schedule received during the direction selection phase.

In the rest of this section, we present four different schemes for assigning the schedule between the different nodes and from/to the coordinator. The different schemes can achieve different fairness-security goals as we quantify in Section V Fairness refers to balancing the access opportunity within nodes and between the from/to coordinator traffic. Therefore, we have four combinations of fairness: node fairness (short and long term) and direction fairness (short and long term). In all schemes, all nodes have to finish one message of transmission before any node can start a new message for fairness purposes. Table II compares the different schemes.

We start by some notations followed by the details of the four schemes.

\section{A. Notations}

The following notations are illustrated in Figure 2

- A session is a group of slots that represent a single selection phase followed by a data transmission phase. The data phase of each session contains $f$ frames.

- A round is defined as a group of $n$ sessions, in which each one of the $n$ nodes is assigned one session.

- A supersession is a group of sessions $(2 n k)$ in which all nodes finish the transmission of one message (i.e. a transmission of $k$ frames in each direction from/to the coordinator).

- A node supersession is a group of $k$ sessions that belong to one node in which this node finishes the transmission of one message.

\section{B. Fair node selection and fair direction division scheme (FN- FD)}

This scheme combines short term node fairness and short term direction fairness (Figure 2(a)). In particular, all nodes must take a turn within the round (in a random fashion) before a node can be assigned another turn by the coordinator. The number of from/to slots within each data transmission session are equal. Therefore, both node and direction distributions have short term fairness. This scheme, however, reduces the ambiguity at the eavesdropper and hence decreases security as we quantify in the next section.
C. Random node selection and fair direction division scheme (RN-FD)

In this scheme (Figure 2(b)), the number of from/to frames within each data session has to be equal (achieving direction fairness). However, the sessions assigned to a specific node can be anywhere within the supersession (random node selection), i.e. there are no rounds.

\section{Fair node selection and random direction division scheme} $(F N-R D)$

In this scheme (Figure 2(c) , each node has to be selected at least once before another node gets a second chance. In other words, each node will take a turn within the round. The direction of traffic from/to the coordinator needn't be balanced within a session, but is balanced on the long term in the supersession.

The send/receive queue at the coordinator though may not be balanced due to the random direction assignment. The constraints of the long term fairness over the direction of traffic increases the amount of state that needs to be kept at the coordinator.

\section{E. Random node selection and random direction division scheme $(R N-R D)$}

In this last scheme, the coordinator divides the sessions among the nodes and the from/to traffic randomly within the supersession. Therefore, both node and direction distributions do not have short term fairness (Figure 2(d)).

This scheme has the advantage of increasing the ambiguity at the eavesdropper and hence increasing security. However, it lacks short term fairness and the coordinator has to keep track of more state for the long term fairness.

\section{ANALYSIS}

In this section, we analyze the different schemes through analysis and simulation in terms of security, overhead, and fairness.

\section{A. Secrecy}

For security, we have two modes, depending on the eavesdropper goal. In the first mode, (single node) the eavesdropper is only interested in the messages of a specific node. The second mode assumes that the eavesdropper is interested in the entire network traffic.

1) Single node attack: Let the selected node for the attack be $s$. We analyze the security of the four different schemes. Note that the total number of slots for all nodes to transmit one message in each direction each is $2 n k$. The corresponding total number of sessions therefore is $\frac{2 n k}{f}$ and the number of sessions allocated to a single node is $\frac{2 k}{f}$.

a) $\boldsymbol{R N}$-FD scheme: In order for Eve to guess the message of $s$, it needs to guess the sessions assigned to $s$. This occurs with probability

$$
=\frac{1}{\left(\begin{array}{c}
\frac{2 n k}{f} \\
\frac{2 k}{f}
\end{array}\right)}
$$


TABLE II

COMPARISON BETWEEN THE DIFFERENT SCHEDULING SCHEMES.

\begin{tabular}{|c|c|c|c|c|c|c|}
\hline Scheme & Abbrev. & $\begin{array}{l}\text { Node } \text { selection } \\
\text { fairness }\end{array}$ & $\begin{array}{l}\text { From/To coordi- } \\
\text { nator (direction) } \\
\text { fairness }\end{array}$ & $\begin{array}{l}\text { One node outage } \\
\text { prob. }\left(\operatorname{Pr}_{\text {Out }}(n)\right)\end{array}$ & $\begin{array}{l}\text { Network } \\
\text { outage prob. } \\
\left(P r_{\text {NOut }}(n)\right)\end{array}$ & Overhead \\
\hline $\begin{array}{l}\text { Random node selection } \\
\text { and random direction divi- } \\
\text { sion }\end{array}$ & $\overline{\text { RN-RD }}$ & Long term & Long term & $\bar{E} \frac{1}{(2 k}$ & \multirow[t]{4}{*}{$\prod_{i=n}^{1} \operatorname{Pr}_{\text {out }}(i)$} & \multirow[t]{4}{*}{$\frac{t(n+f)}{f l+t(n+f)}$} \\
\hline $\begin{array}{l}\text { Random node selection } \\
\text { and fair direction division }\end{array}$ & RN-FD & Long term & Short term & $\frac{1}{\left(\begin{array}{c}\frac{2 n k}{f} \\
\frac{2 k}{f}\end{array}\right)} \frac{1}{\left(\begin{array}{l}f \\
\frac{f}{2}\end{array}\right)^{\frac{2 k}{f}}}$ & & \\
\hline $\begin{array}{l}\text { Fair node selection and } \\
\text { random direction division }\end{array}$ & FN-RD & Short term & Long term & $\left(\frac{1}{n}\right)^{\frac{2 k}{f}} \frac{1}{\left(\begin{array}{c}2 k \\
k\end{array}\right)}$ & & \\
\hline $\begin{array}{l}\text { Random within round } \\
\text { node selection and fair } \\
\text { direction division }\end{array}$ & FN-FD & Short term & Short term & $-\frac{1}{(2 k)}$ & & \\
\hline
\end{tabular}

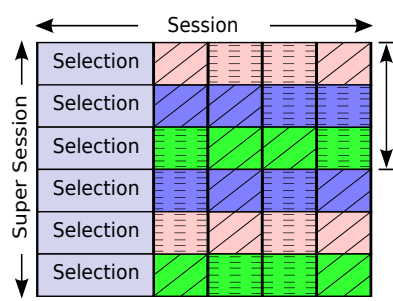

Fair node selection and fair direction division (FN-FD)

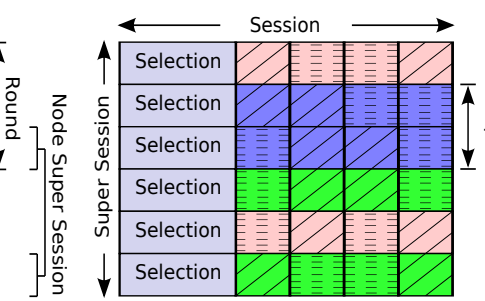

b) Random node selection
direction division (RN-FD)
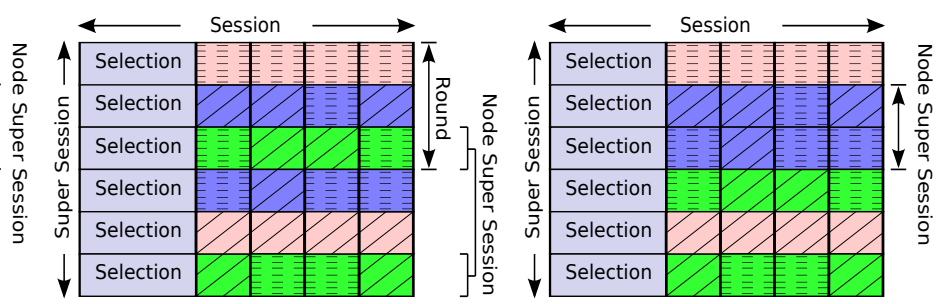

tion division (FN-RD).

dom direction division (RN-RD).

Fig. 2. Four different schemes for two-way communication. The direction selection phase between the selection and data transmission phase is not shown for clarity. Different colors represent different nodes while different hashing pattern represent the from/to slots.

In addition, Eve has to guess the direction of the frames to avoid mixing the packets from/to the coordinator. This occurs with probability:

$$
=\frac{1}{\left(\begin{array}{c}
f \\
\frac{f}{2}
\end{array}\right)}
$$

Therefore, the outage probability for this scheme, for an $n$-node network $\left(\operatorname{Pr}_{\text {out }}(n)\right)$ is:

$$
P r_{\text {out }}(n)=\frac{1}{\left(\begin{array}{l}
\frac{2 n k}{f} \\
\frac{2 k}{f}
\end{array}\right)} \frac{1}{\left(\begin{array}{l}
f \\
\frac{f}{2}
\end{array}\right)^{\frac{2 k}{f}}}
$$

b) $\boldsymbol{F N}$-RD scheme: In this case, Eve needs to decide in each round which session belongs to $s$. Therefore the probability of correctly guessing Eve's sessions in the entire supersession is:

$$
=\left(\frac{1}{n}\right)^{\frac{2 k}{f}}
$$

Once the sessions of node $s$ are determined, Eve has to guess which of the total of $2 k$ frames are to the coordinator and which are from it. This occurs with probability:

$$
=\frac{1}{\left(\begin{array}{c}
2 k \\
k
\end{array}\right)}
$$

Therefore, the outage probability is this case is

$$
P r_{\text {out }}(n)=\left(\frac{1}{n}\right)^{\frac{2 k}{f}} \frac{1}{\left(\begin{array}{c}
2 k \\
k
\end{array}\right)}
$$

c) $\boldsymbol{R N}$-RD scheme: Similar to equations 2 and 6, the outage probability in this case is:

$$
P r_{\text {out }}(n)=\frac{1}{\left(\begin{array}{c}
\frac{2 n k}{f} \\
\frac{2 k}{f}
\end{array}\right)} \frac{1}{\left(\begin{array}{c}
2 k \\
k
\end{array}\right)}
$$

d) FN-FD scheme: Similarly, the outage probability here can be obtained by combining equations 3 and 5 as:

$$
\operatorname{Pr}_{\text {out }}(n)=\left(\frac{1}{n}\right)^{\frac{2 k}{f}} \frac{1}{\left(\begin{array}{l}
f \\
\frac{f}{2}
\end{array}\right)^{\frac{2 k}{f}}}
$$

2) Network-wide attack: In this attack, Eve is interested in obtaining the entire network traffic. Once Eve guesses the frames of one node, the problem size decreases to that of an $n-1$ node network. Therefore, the outage probability in this case $\left(P r_{\text {NOut }}\right)$ is:

$$
\operatorname{Pr}_{\mathrm{NOut}}=\prod_{i=n}^{1} \operatorname{Pr}_{\mathrm{out}}(i)
$$

\section{B. Overhead}

The four different schemes have the same overhead which is due to the selection and direction determination phases. Therefore, the overhead for all four schemes is:

$$
\frac{t(n+f)}{f l+t(n+f)}
$$




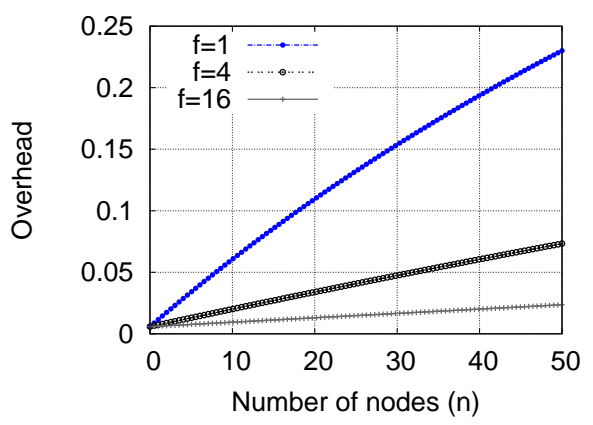

Fig. 4. Effect of different parameters on overhead $(l=1024$ bits, $t=6)$. Note that a longer frame length $(l)$ leads to lower overhead.

which is a function of the number of nodes $(n)$, dialog codes preamble length $(t)$, number of slots in a session $(f)$, and the data frame length $(l)$.

\section{Numerical Results}

1) Secrecy analysis: Figure 3 shows the effect of changing the system parameters, i.e. $n, k$, and $f$, on the outage probability for the four schemes. The figure shows that all schemes have the advantage of enhancing the secrecy with the increase of the number of nodes in the network. Increasing $k$ increases the space of guessing at Eve, and hence enhances secrecy. Increasing $f$ leads to increasing the length of the data transmission phase and hence reducing the frequency of the selection phase. This reduces secrecy.

The figure also shows that the RN-RD scheme has the highest secrecy. This is due to the increased ambiguity at Eve due to the randomization of both node selection and direction. On the other extreme, the FN-FD scheme has the least secrecy. The other two schemes have a secrecy outage probability in between: As the data phase length increases, direction randomization leads to more secrecy than node randomization.

2) Overhead: Figure 4 shows the effect of changing the system parameters on the system overhead. The figure shows that the overhead increases with the increase of the number of nodes in the network and the decrease of the data transmission phase length. Therefore, a trade-off exists between overhead and secrecy. The operation point can be selected based on the specific application need.

3) Fairness: For the fairness in node selection, we use the variance of the difference between two consecutive sessions indices as our metric. The more consistent this difference, the lower the variance, and the higher the fairness. More formally, if the session indices assigned to a node are $\left\{s_{i} \| 0<i<\right.$ $2 k / f\}$, then $d_{i}=s_{i+1}-s_{i}$ and the unfairness index equals $\operatorname{Var}\left(d_{i}\right)$. Figure 5 shows the effect of the different parameters on node fairness. The figure confirms that the round-based schemes are fairer than the random schemes. As the number of nodes $(n)$ increases, the unfairness increases. On the other hand, for a fixed $n=8$, increasing the number of sessions, by either increasing $k$ or reducing $f$, the unfairness increases. However, this is limited to within a round in the short term node fairness (FN) schemes and is more variable in the long

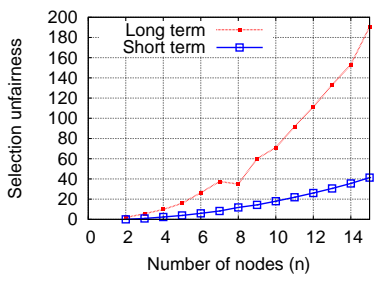

(a) Num. of nodes

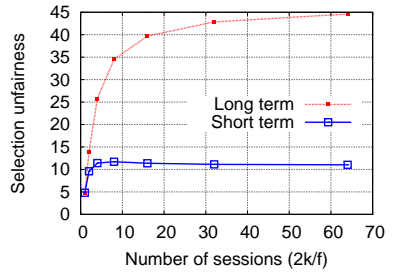

(b) Num. of sessions
Fig. 5. Effect of different parameters on node fairness.

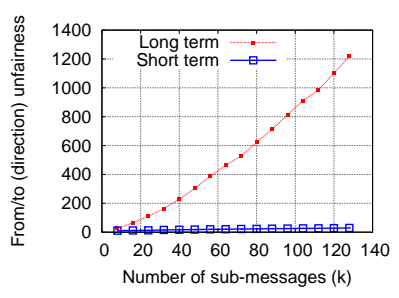

(a) Num. of sub-messages

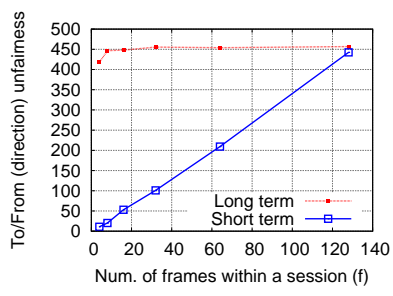

(b) Session size
Fig. 6. Effect of different parameters on direction fairness.

term node fairness schemes. The saturation in both cases is due to the limitation imposed by the supersession size.

For direction fairness, our metric is the absolute difference between the sum of the send and receive indices within a specific node supersession, averaged over all nodes. The smaller this number, the higher the fairness. Note that since the fairness metric is node based, it is independent from the number of nodes $n$.

Figure 6 shows the effect of the different parameters on direction fairness. The figure confirms that the short term direction fairness (FD) schemes are fairer than the long term direction fairness (RD) schemes. As the number of frames required to construct a mesage $(k)$ increases, the unfairness increases in the long term direction fairness scheme as the overall number of slots in the node supersession will increase. $k$ has no effect on the short term direction fairness schemes as all direction selections are based on a round, which is independent of $k$. This is the opposite case as we fix $k$ and change the number of frames within a session $(f)$. In this case, the performance of the completely random case is independent of the number of frames within a session, as all sessions are concatenated in one supersession. Increasing $f$ increases the unfairness of the short term direction fairness schemes. However, their worst case performance is bounded by the performance of the long term direction fairness scheme, where on session becomes a node supersession.

4) Comparison with a traditional two-node system: Figure 7 compares the proposed schemes to the practical provably secure two-node scheme proposed in [11] under typical parameters for all schemes. The scheme in [11] is based on randomization between two nodes. A direct extension for this case to the multi-node case is to apply it pairwise to each transmitter receiver. The figure shows that this reduces secrecy significantly, with several orders of magnitude and this loss in secrecy increases with the increase in the number of nodes. 


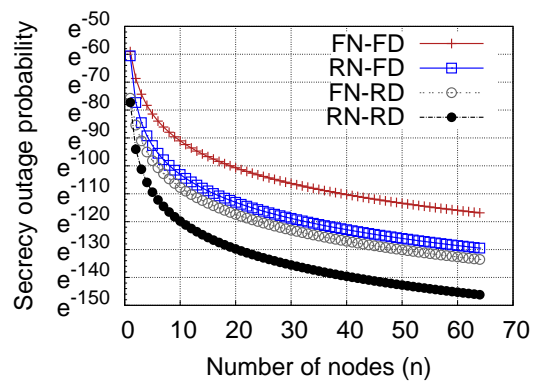

(a) Num. of nodes

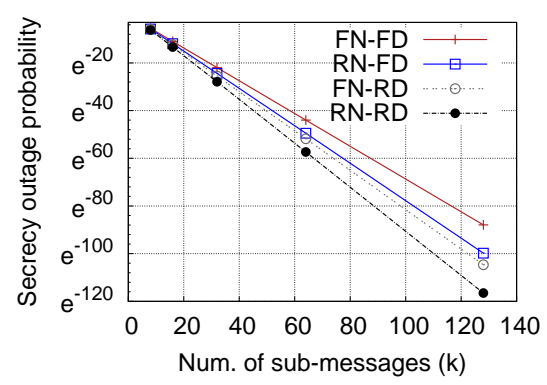

(b) Num. of frames/message

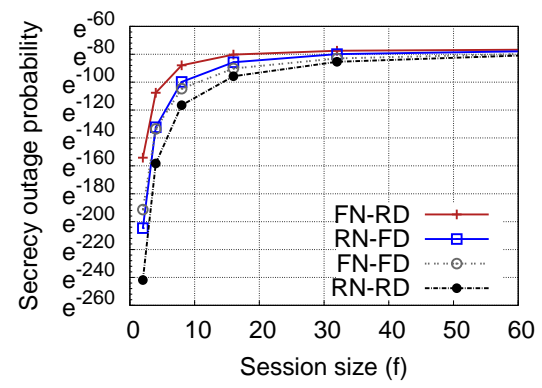

(c) Session length

Fig. 3. Effect of different parameters on secrecy.

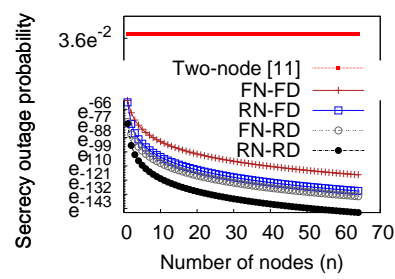

(a) Secrecy

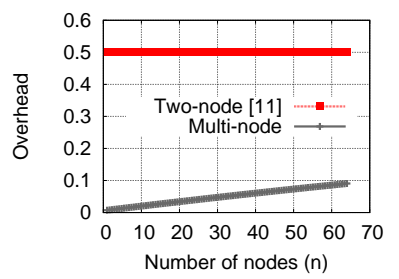

(b) Overhead
Fig. 7. Comparison with previous two-node based practical provably secure schemes [11].

Since [11] does not leverage the multi-node in its design, both its security and overhead is independent of $n$. Our proposed schemes also have much better overhead under typical network sizes.

\section{System Implementation}

We have also implemented the proposed scheme on TelosB motes equipped with CC2420 radio chips which come with half-duplex antennas. The motes run the TinyOS operating system.

The network consists of three types of nodes nodes:

1) An observer node, which plays a double role in our setup. First, it plays the role of the passive eavesdropper which sniffs all sent frames. Second, it is responsible for synchronizing all nodes in the network by sending a pulse (synchronization frame in our case) at constant intervals to initiate the start of a slot and hence transmission of frames. The remaining nodes react to these synchronization frames.

2) A normal node (representing one of the $n$ legitimate nodes).

3) A coordinator node, which selects which node to transmit and the direction of traffic.

The implementation results confirm the analysis results in the previous sections. More details about the implementation can be found in [15].

\section{CONCLUSION}

We presented a novel practical and provably secure solution to the multi-node wireless communication problem. Our solution is based on hiding the identity of the communicating nodes from the eavesdropper. We presented four different variations of the basic scheme that can achieve different fairnesssecurity tradeoffs. We evaluated the proposed techniques using analysis and implementation. Our results show that our scheme outperforms direct extensions of the two-node communication schemes in terms of both overhead and secrecy, highlighting its suitability for highly secure applications.

\section{REFERENCES}

[1] R. Keon, "RSA Laboratories' Frequently Asked Questions About Today's Cryptography, Version 4.1," Published online: http://www rsa. com/rsalabs/faq/February, 2001.

[2] C. E. Shannon, "Communication theory of secrecy systems," Bell System Technical Journal, vol. 28, pp. 656-715, 1949.

[3] A. D. Wyner, "The wire-tap channel," Bell Systems Technical Journal, vol. 54, no. 8, pp. 1355-1387, January 1975.

[4] U. Maurer, "Secret key agreement by public discussion from common information," IEEE Trans. on Information Theory, vol. 39, no. 3, pp. 733-742, may 1993.

[5] P. K. Gopala, L. Lai, and H. E. Gamal, "On the secrecy capacity of fading channels," IEEE Trans. on Information Theory, 2006.

[6] X. Tang, R. Liu, and P. Spasojevic, "On the achievable secrecy throughput of block fading channels with no channel state information at transmitter," in CISS. IEEE, 2007, pp. 917-922.

[7] Y. Omar, M. Youssef, and H. El Gamal, "ARQ secrecy: From theory to practice," in IEEE Information Theory Workshop ITW 2009, October 2009, pp. 6-10.

[8] M. Elsabagh, Y. Abdallah, M. Youssef, and H. El Gamal, "ARQ security in Wi-Fi and RFID networks," in Forty-Eighth Annual Allerton Conference, September 2010.

[9] M. Elsabagh, M. Youssef, and H. El Gamal, "ARQ security in rfid networks," in IEEE ICC '11 Workshop on Physical Layer Security, June 2011.

[10] Y. Omar, M. Abdelatif, M. Youssef, A. Sultan, and H. Elgamal, "Keys through ARQ: Theory and practice," in IEEE Transactions on Information Forensics \& Security, To appear.

[11] A. Elmorsy, M. Yasser, M. Elsabagh, and M. Youssef, "Practical Provably Secure Communication for Half-Duplex Radios," in Соттиnications (ICC), 2011 IEEE International Conference on. IEEE, 2011, pp. $1-5$.

[12] A. Arora, "Dialog codes for secure wireless communications," Processing in Sensor Networks, 2009. IPSN, 2009.

[13] K. Khalil, O. O. Koyluoglu, H. E. Gamal, and M. Youssef, "Opportunistic secrecy with a strict delay constraint," IEEE Transactions on Communications, to appear.

[14] A. El Gamal, O. Koyluoglu, M. Youssef, and H. El Gamal, "Achievable secrecy rate regions for the two-way wiretap channel," IEEE Transactions on Information Theory, to apper.

[15] O. Hassan, M. Fouad, and M. Youssef, "Demonstrating practical provably secure multi-node communication," in Proceedings of the The Seventh ACM International Workshop on Wireless Network Testbeds, Experimental evaluation, and Characterization (WiNTECH'12), in conjunction with MobiCom 2012, 2012. 